\title{
A Generic NeUral NeTWORK APPROACH TO INFER SEgmenting Classifiers For Disease-Associated REGIONS IN MEDICAL IMAGES
}

\section{A PREPRINT}

\author{
David Schuhmacher ${ }^{1,2}$
}

Klaus Gerwert $^{1,3}$

Axel Mosig ${ }^{1,2}$

${ }^{1}$ Center for Protein Diagnostics

Ruhr-Universität Bochum

Gesundheitscampus 4

44801 Bochum, Germany

axel.mosig@rub.de

\author{
${ }^{3}$ Faculty of Biology and Biotechnology \\ Chair of Biophysics \\ Gesundheitscampus 4 \\ 44801 Bochum, Germany
}

February 25, 2020

\begin{abstract}
In many settings in digital pathology or radiology, it is of predominant importance to train classifiers that can segment disease-associated regions in medical images. While numerous deep learning approaches, most notably U-Nets, exist to learn segmentations, these approaches typically require reference segmentations as training data. As a consequence, obtaining pixel level annotations of histopathological samples has become a major bottleneck to establish segmentation learning approaches. Our contribution introduces a neural network approach to avoid the annotation bottleneck in the first place: our approach requires two-class labels such as cancer vs. healthy at the sample level only. Using these sample-labels, a meta-network is trained that infers a segmenting neural network which will segment the disease-associated region (e.g. tumor) that is present in the cancer samples, but not in the healthy samples. This process results in a network, e.g. a $U$-Net, that can segment tumor regions in arbitrary further samples of the same type.

We establish and validate our approach in the context of digital label-free pathology, where hyperspectral infrared microscopy is used to segment and characterize the disease status of histopathological samples. Trained on a data set comprising infrared microscopic images of 100 tissue microarray spots labelled as either cancerous or cancer-free, the approach yields a $U$-Net that reliably identifies tumor regions or the absence of tumor in an independent test set involving 40 samples.

While our present work is focused on training a $U$-Net for infrared microscopic images, the approach is generic in the sense that it can be adapted to other image modalities and essentially arbitrary segmenting network topologies.
\end{abstract}

Keywords: Image Segmentation, Deep Learning, Segmentation-free training 
A Generic Neural Network Approach to Infer Segmenting Classifiers for Disease-Associated Regions in Medical Images

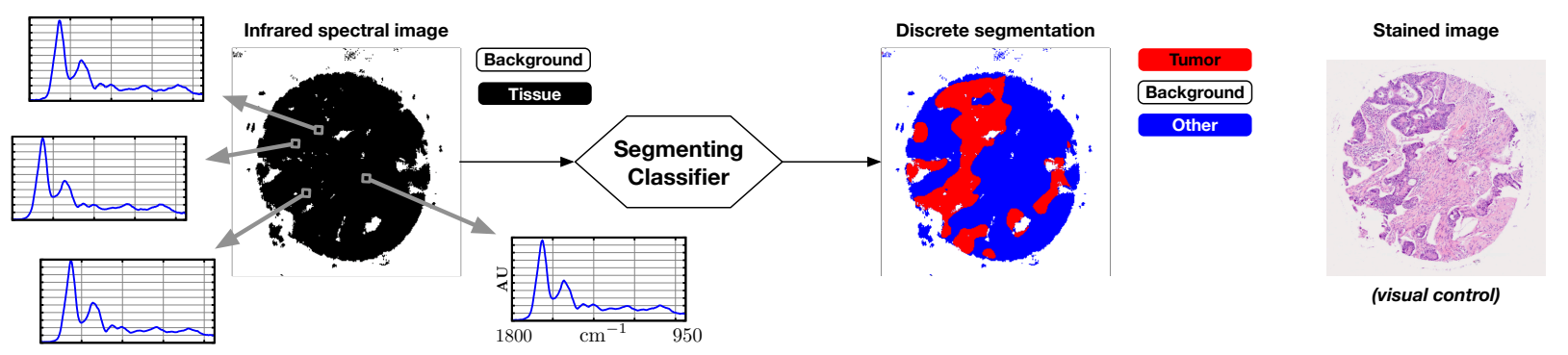

Figure 1: Principle of label-free digital pathology. The infrared microscopic image is acquired from an unstained sample. Although the differences between different pixel spectra are barely visible by eye (Supplementary Figure S1), it is well-established that they are highly representative for and can thus differentiate different tissue components or disease status [1, 5, 7, 11]. The spectral image can thus be used to segment disease-associated regions as illustrated, or resolve even further details of the tissue architecture [1,11]. Our contribution deals with obtaining classifiers that can identify one disease-associated component such as tumor. In the context of label-free digital pathology, hematoxylin and eosin (H\&E) stained images (right) are displayed mainly for visual reference and control.

\section{Introduction}

The annotation of disease-related regions is a major bottleneck for adapting machine learning in biomedical imaging. Annotations are crucially important to train segmenting classifiers, whose relevance for medical imaging has been well realized since the introduction of U-Nets [17] and SegNets [3], not least due to the visual interpretability of segmentations by humans. However, training such segmenting classifiers requires large amounts of precise reference segmentations. Correspondingly, enormouse efforts have been made to obtain reference annotations, making annotation a labor-intensive and costly bottleneck.

As a recent and prominent example, the Gland Segmentation in Colon Histology Images challenge [19] provided a significant number of high-quality reference segmentations performed by a pathologist as ground truth and training data. More recently, the authors of [9] and [2] introduced crowd-based bioimage annotation systems, while the authors in [1] use an image annotation tool to obtain ground truth annotations for different components of lung tissue in 388 sample spots.

Overcoming the annotation bottleneck is particularly challenging in the context of infrared microscopic images. Infrared microscopy provides hyperspectral images of tissue samples at high spatial resolution, making it an ideal tool for resolving the tissue structure of histopathological samples and characterizing their disease status (Fig. 1). Infrared microscopy requires no staining prior to spectral image acquisition, and has been applied succesfully as a label-free digital pathology approach in increasingly large clinical studies which tackled diverse pathology related questions such as identifying tumors [13], grading colon carcinoma [11, 12] or identifying subtypes of lung carcinoma [5, 7, 1].

Our contribution aims to overcome the annotation bottleneck through an approach which trains an arbitrary segmenting neural network using annotations at the sample level only. More specifically, we assume that each sample is annotated as either a disease class such as cancer or a healthy class such as cancer free. Furthermore, we assume that each disease image contains a disease-specific region that is not contained in the healthy control samples - for example, cancer samples contain a certain fraction of tumor, while cancer free spots do not contain tumor. The goal of our newly proposed CompSegNet approach is to train a segmenting classifier that will segment this disease-specific region.

In medical image analysis, a major advantage of segmenting networks over merely classifying networks such as plain convolutional neural networks (CNNs) is that they explicitely locate disease-specific regions, so that their output is interpretable by humans, while merely classifying CNNs are black boxes which do not provide information about the grounds on which an image was assigned to a particular class [15]. As surveyed in [8], the problem of interpreting CNN models has attracted major attention, but has been adressed in a rather post-mortem manner: A CNN is first being trained, and seeking interpretable evidence is performed only after training the network. This has been investigated using a plethora of approaches. Saliency maps [18], for example, are applicable if the images to be classified can be aligned into a unique coordinate system, as applied recently for Alzheimer's diease related whole-brain PET scans [6]. In the majority of medical image analysis tasks in radiology or pathology, where images are not alignable, techniques such as class activation maps (CAMs) [21] can be applied, which have been utilized recently in medical image analysis [20]. 
A Generic Neural Network Approach to Infer Segmenting Classifiers for Disease-Associated Regions in Medical Images

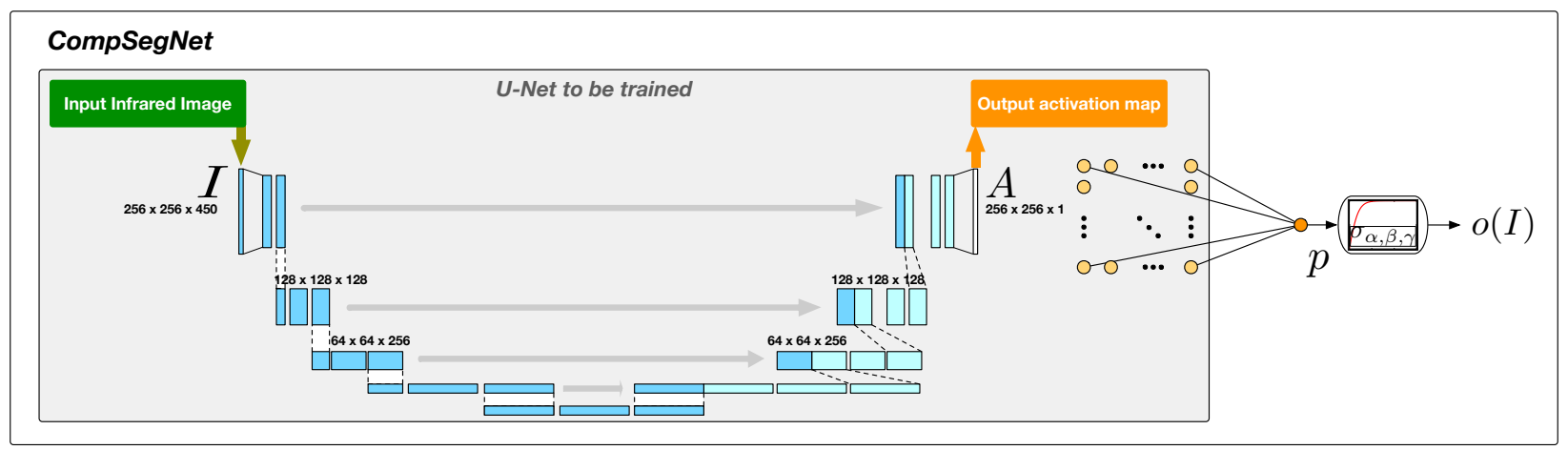

Figure 2: The CompSegNet is a simple extension of a segmenting neural network by a pooling neuron $p$, as described in Section 3.1

Viewed from the perspective of model interpretation, our approach shifts the interpretation problem from post-mortem to the level of training: Rather than seeking interpretable location-specific evidence in a readily trained model, we hardwire interpretability into the target function, and the process of training optimizes interpretability of the segmentations obtained from the classifier.

\section{Infrared Imaging Data}

We use an infrared image dataset of three tissue microarray (TMA) slides involving colon carcinoma related samples. The TMA slides CO1002b, CO722 and BC051111 were purchased from US Biomax Inc., MD, USA, each comprising 100-200 circular spots of tissue samples obtained from more than 50 different patients. Each spot has a diameter of roughly $1 \mathrm{~mm}$. Infrared pixel spectra were preprocessed using resonant Mie correction following the approach from [4], yielding the spectrum in the range between 1800-948 $\mathrm{cm}^{-1}$ represented as a 450 dimensional vector. Each spot is represented as a $256 \times 256$ spectral image. Spectra at pixel positions not covered by sample were masked as background following commonly established procedures [11]. Spot labels were obtained from the annotations accompanying the data sets 1 , which classify each spot into either Malignant tumor (T), Malignant tumor (stage I-III) (TI-III), Metastasis (M), Adjacent normal colon tissue (NAT), or Normal tissue (N). Following the terminology of [16], we divided the data set into three parts: 100 of the spots were selected as training set, seven spots as validation set and further fourty spots as an independent test set. Training and validation data were selected from arrays $C O 1002 b$ and $C O 722$, while the independent test set was selected from $B C 051111$. The latter array contains 180 cancer spots and 20 healthy control spots. To obtain a largest possible balanced independent test test, 20 cancer spots were chosen arbitrarily to match the 20 healthy controls, yielding our 40 spots as independent test set. In all three arrays, spots labelled as T, TI-III or M were used as cancer spots; cancer-free spots were selected only from those labelled as $\mathbf{N}$.

\section{Approach}

\subsection{Network Topology}

The basic idea of our approach is to extend a segmenting neural network by accumulating the pixel activations in the output layer in a pooling neuron $p$ as displayed in Figure 2. We will refer to this extended network as a comparative segmentation network or CompSegNet for short. The specific CompSegNet used throughout the paper is based on the original $U$-Net topology, except the number of input channels has been extended to 450 to accomodate infrared images as input. The goal of the CompSegNet is to train the underlying U-Net in a weakly supervised manner. To this end, we assume that each pixel of the activation map is bounded within the interval $[0,1]$, e.g. by a sigmoid transfer function, so that the pooling neuron $p$ will "count" the number of active pixels. With $p$ counting active pixels, we can specify the target function: If an input image $I^{-}$is labeled as cancer-free, there should be zero pixels recognized as tumor, in other words, the output $o\left(I^{-}\right)$of the CompSegNet should be zero. Conversely, if an input image $I^{+}$is labeled as cancer, there should be a significantly large number of pixels in the activation map whose activation is close to one. In other words, $o\left(I^{+}\right)$should be as large as possible.

\footnotetext{
${ }^{1}$ https://www.biomax.us/tissue-arrays/Colon
} 
A Generic Neural Network Approach to Infer Segmenting Classifiers for Disease-Associated Regions in Medical Images

\subsection{Loss Function}

To achieve the desired effect of training the CompSegNet, we design a loss function for the pooling neuron $p$ that maximizes the pooled activation from the cancer images while minimizing the pooled activation from the cancer-free images. We assume our training data consist of spectral images $I_{1}, \ldots, I_{M}$ along with labels $\ell(1), \ldots, \ell(M)$, where $\ell(j)=1$ if $I_{j}$ is a cancer image and $\ell(j)=0$ if $I_{j}$ is a cancer-free image. Furthermore, we assume the transfer function $\sigma_{p}$ of the pooling neuron $p$ is a sigmoid-like function with an upper bound of $\alpha$, i.e., $\sigma_{p}(z) \leq \alpha$ for all $z$, as well as $\sigma_{p}(0)=0$. After applying this transfer function, the output $o\left(I_{j}\right)$ of applying the CompSegNet output to image $j$ should satisfy the following properties:

(L1) If $\ell(j)=1$, then $o\left(I_{j}\right) \rightarrow \alpha$, i.e., in a cancer image, a significantly large number of tumor pixels should be identified.

(L2) If $\ell(j)=0$, then $o\left(I_{j}\right) \rightarrow 0$, i.e., in a cancer-free image, no tumor pixels should be identified.

Thus, defining the loss function $g$ as the root-mean-squared error

$$
g(I, \ell)=\sum_{j}\left|o\left(I_{j}\right)-\alpha \ell(j)\right|,
$$

will optimize towards properties (L1) and (L2) being satisfied.

The transfer function $\sigma_{p}$ for the pooling neuron $p$ needs some attention. In terms of the tumor regions to be identified, a plain sigmoid function translates to the unrealistic assumption that an "ideal" tumor sample consists of $100 \%$ tumor. In reality, it may be more realistic to assume that cancerous samples contain more than $10 \%$ tumor tissue - a value at which the sigmoid function has a steep gradient, so that a plain sigmoid target function may cause poor convergence when training the CompSegNet. We thus introduce a modified transfer function

$$
\sigma_{\alpha, \beta, \gamma}(x)=\alpha \cdot\left(2 \cdot(1+\exp (-\beta x / \alpha))^{-1}-1\right)+\gamma x
$$

involving three parameters $\alpha, \beta$ and $\gamma$. Parameter $\alpha$ specifies the minimum percentage of tumor in the cancer samples, $\beta$ is a scaling paramter, and $\gamma$ specifies a typically small asymptotic linear gain whose purpose is to avoid the vanishing gradient problem and introduce an incentive to detect more tumor in cancer samples where more significantly more than $10 \%$ of the tissue is tumor. All our experiments were conducted using fixed parameters $\alpha=.1, \beta=5$ and $\gamma=.001$.

With the transfer and loss functions introduced above, training the CompSegNet will train the underlying $U$-Net to identify as much cancer as possible in the tumor-samples, while identifying as little tumor as possible in the tumor-free control samples. Put into even simpler terms, the CompSegNet is designed in a way such that training the CompSegNet forces the underlying $U$-Net to learn to identify the tumor regions. After convergence of the CompSegNet training on a sufficiently large number of images using backpropagation, the CompSegNet itself is no longer needed, and only the underlying $U$-Net is extracted and can serve as a readily trained segmenting network that can identify tumor regions.

Implementation. We trained the network described above using RMSprop with a mini-batch size of $k=7$ and a learning rate of .001. Pooling neurons $p^{+}$and $p^{-}$use the modified sigmoid activation function $\sigma_{\alpha, \beta, \gamma}$; activation map neurons $a^{+}$and $a^{-}$use a standard sigmoid activation. All other neurons use the SoftPlus activation function. All neural networks were implemented in tensorflow.

\section{Results}

We evaluated our approach on the data set described in Section 2 using the network topology described in Section 3.1 using a mini-batch size of $k=7$ and starting from random initialization. We ran 400 epochs on the test data set comprising 100 samples and selected the model with minimal loss on the seven samples from validation data set (epoch 385), requiring roughly three days of wall clock computation time using four NVidia GeForce 1080Ti graphics cards with a total of 44 GB of GPU memory. In the activation maps of the validation samples, a threshold was visually identified to seperate tumor from non-tumor. One and the same threshold $(.35 \cdot 255)$ was applied to activation maps of 20 cancer and 20 cancer-free spots in the independent test set from TMA from slide BC051111. By varying a percentage threshold for relative tumor content to classify spots as cancer or cancer-free, we obtained a ROC curve with an AUC of 93.25\% (Supplementary Figure S3). The agreement between the activation maps and tumor is displayed in Fig. 3 and in Supplementary Figure S2. The agreement is further supported by an average Dice score of $\mu=.42(\sigma=.19)$ between the thresholded activation maps and manual ground truth annotation by an independent expert for the 20 cancer spots underlying the ROC curve. 
medRxiv preprint doi: https://doi.org/10.1101/2020.02.27.20028845; this version posted February 29, 2020. The copyright holder for this preprint (which was not certified by peer review) is the author/funder, who has granted medRxiv a license to display the preprint in perpetuity. All rights reserved. No reuse allowed without permission.

A Generic Neural Network Approach to Infer Segmenting Classifiers for Disease-Associated Regions in Medical Images A PREPRINT
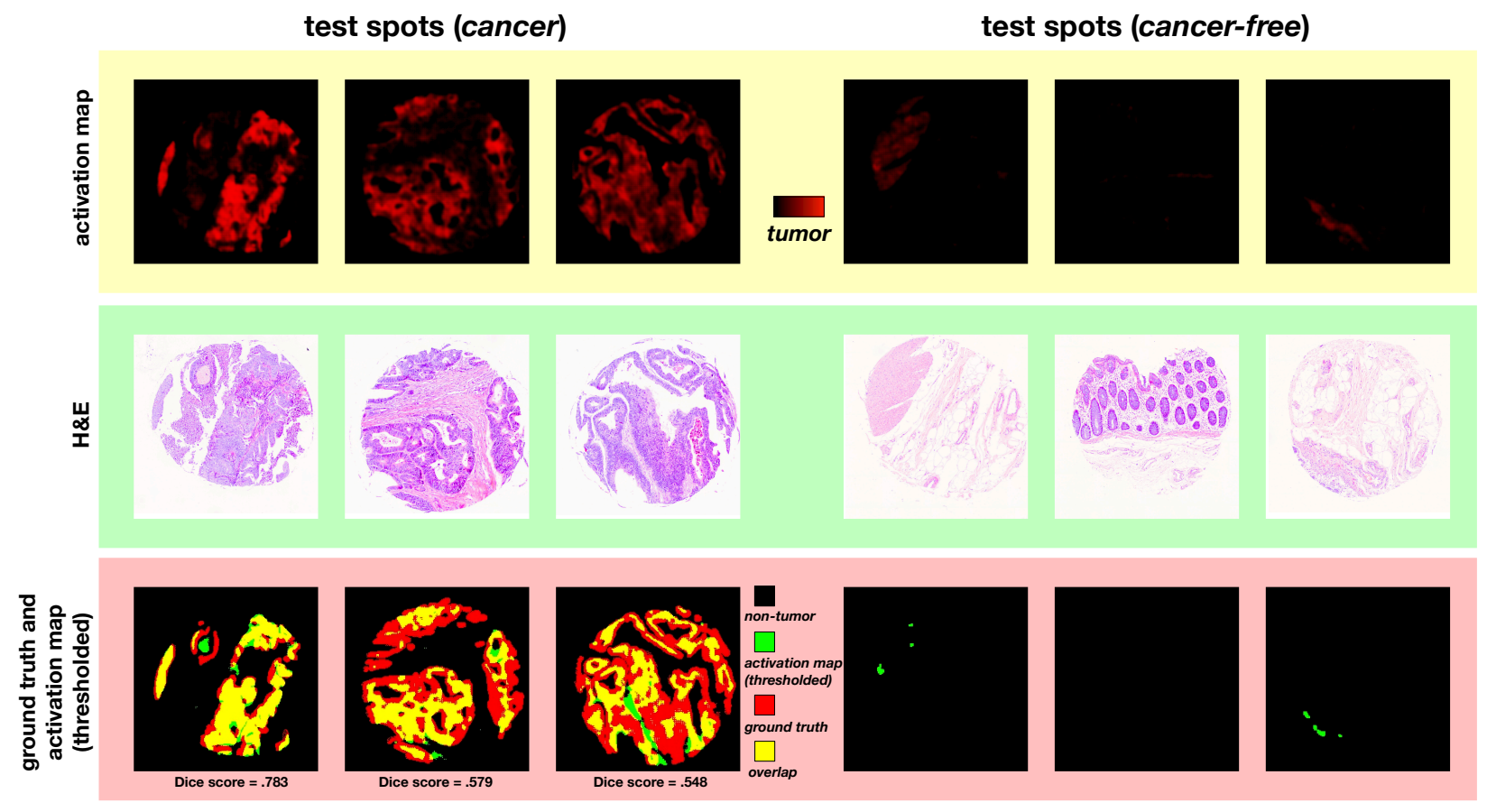

Figure 3: Segmentations of six spots of the independent test data set obtained using the CompSegNet. Tumor regions in the three cancer spots agree well with the conventional staining images. Among the three cancer-free spots, only small amounts of false positive tumor are being detected. The complete balanced independent test set is displayed in Supplementary Figure 2.

\section{Discussion}

Our results provide proof-of-concept that segmenting classifiers can be trained on a relatively small infrared imaging data set using sample labels only, and we could demonstrate that the resulting classifier generally works on independent validation samples, despite the tissue microarray data set which certainly exhibits a higher level of heterogeneity than a typical clinical study.

Due to the generic nature of the remarkably simple CompSegNet approach, it suggests itself to validate the CompSegNet on other imaging modalities in pathology or radiology as well as on other network topologies. The approach is particularly attractive for hyperspectral modalities including variants of Raman microscocopy [14] where multidimensional imaging data inherently defy visual inspectability. For infrared microscopy driven label-free digital pathology, this immediately relieves established workflows [1, 10] from their reliance on segmentation annotations, which predominantely rely on conventional histopathological staining. It may thus infer regions that are causally linked to phenotype status that can only be determined at the whole-sample or even patient level such as responder vs. non-responder, or when contrasting differences in mutation type. It will also be relevant to investigate convergence of the CompSegNet, which may be improved using other target functions and other segmenting networks. Furthermore, investigating the properties of the CompSegNet with respect to model interpretation will be of high relevance, as well as systematically assessing how far the trained models generalize beyond the given training and validation data.

\section{References}

[1] A. Akalin, X. Mu, M. A. Kon, A. Ergin, S. H. Remiszewski, C. M. Thompson, D. J. Raz, and M. Diem. Classification of malignant and benign tumors of the lung by infrared spectral histopathology (SHP). Laboratory investigation, 95(4):406, 2015.

[2] M. Amgad, H. Elfandy, H. H. Khallaf, L. A. Atteya, M. A. Elsebaie, L. S. A. Elnasr, R. A. Sakr, H. S. Salem, A. F. Ismail, A. M. Saad, et al. Structured crowdsourcing enables convolutional segmentation of histology images. Bioinformatics, 2019. 
medRxiv preprint doi: https://doi.org/10.1101/2020.02.27.20028845; this version posted February 29, 2020. The copyright holder for this preprint (which was not certified by peer review) is the author/funder, who has granted medRxiv a license to display the preprint in perpetuity. All rights reserved. No reuse allowed without permission.

A Generic Neural Network Approach to Infer Segmenting Classifiers for Disease-Associated Regions in Medical Images A PREPRINT

[3] V. Badrinarayanan, A. Kendall, and R. Cipolla. Segnet: A deep convolutional encoder-decoder architecture for image segmentation. IEEE transactions on pattern analysis and machine intelligence, 39(12):2481-2495, 2017.

[4] P. Bassan, A. Kohler, H. Martens, J. Lee, H. J. Byrne, P. Dumas, E. Gazi, M. Brown, N. Clarke, and P. Gardner. Resonant mie scattering (RMieS) correction of infrared spectra from highly scattering biological samples. Analyst, 135(2):268-277, 2010.

[5] B. Bird, M. Miljkovic, S. Remiszewski, A. Akalin, M. Kon, and M. Diem. Infrared spectral histopathology (SHP): a novel diagnostic tool for the accurate classification of lung cancer. Laboratory investigation, 92(9):1358, 2012.

[6] Y. Ding, J. H. Sohn, M. G. Kawczynski, H. Trivedi, R. Harnish, N. W. Jenkins, D. Lituiev, T. P. Copeland, M. S. Aboian, C. Mari Aparici, et al. A deep learning model to predict a diagnosis of alzheimer disease by using 18F-FDG PET of the brain. Radiology, 290(2):456-464, 2018.

[7] F. Großerueschkamp, A. Kallenbach-Thieltges, T. Behrens, T. Brüning, M. Altmayer, G. Stamatis, D. Theegarten, and K. Gerwert. Marker-free automated histopathological annotation of lung tumour subtypes by FTIR imaging. Analyst, 140(7):2114-2120, 2015.

[8] R. Guidotti, A. Monreale, S. Ruggieri, F. Turini, F. Giannotti, and D. Pedreschi. A survey of methods for explaining black box models. ACM Computing Surveys (CSUR), 51(5):93, 2018.

[9] A. J. Hughes, J. D. Mornin, S. K. Biswas, L. E. Beck, D. P. Bauer, A. Raj, S. Bianco, and Z. J. Gartner. Quanti.US: a tool for rapid, flexible, crowd-based annotation of images. Nature methods, 15(8):587, 2018.

[10] A. Kallenbach-Thieltges, F. Großerüschkamp, A. Mosig, M. Diem, A. Tannapfel, and K. Gerwert. Immunohistochemistry, histopathology and infrared spectral histopathology of colon cancer tissue sections. Journal of biophotonics, 6(1):88-100, 2013.

[11] C. Kuepper, F. Großerueschkamp, A. Kallenbach-Thieltges, A. Mosig, A. Tannapfel, and K. Gerwert. Label-free classification of colon cancer grading using infrared spectral histopathology. Faraday discussions, 187:105-118, 2016.

[12] C. Kuepper, A. Kallenbach-Thieltges, H. Juette, A. Tannapfel, F. Großerueschkamp, and K. Gerwert. Quantum cascade laser-based infrared microscopy for label-free and automated cancer classification in tissue sections. Scientific reports, 8(1):7717, 2018.

[13] P. Lasch, W. Haensch, D. Naumann, and M. Diem. Imaging of colorectal adenocarcinoma using FT-IR microspectroscopy and cluster analysis. Biochimica et Biophysica Acta Molecular Basis of Disease, 1688(2):176-186, 2004.

[14] D. A. Orringer, B. Pandian, Y. S. Niknafs, T. C. Hollon, J. Boyle, S. Lewis, M. Garrard, S. L. Hervey-Jumper, H. J. Garton, C. O. Maher, et al. Rapid intraoperative histology of unprocessed surgical specimens via fibre-laser-based stimulated Raman scattering microscopy. Nature biomedical engineering, 1(2):0027, 2017.

[15] W. N. Price. Big data and black-box medical algorithms. Science translational medicine, 10(471):eaao5333, 2018.

[16] B. D. Ripley and N. Hjort. Pattern recognition and neural networks. Cambridge university press, 1996.

[17] O. Ronneberger, P. Fischer, and T. Brox. U-net: Convolutional networks for biomedical image segmentation. In International Conference on Medical image computing and computer-assisted intervention, pages 234-241. Springer, 2015.

[18] K. Simonyan, A. Vedaldi, and A. Zisserman. Deep inside convolutional networks: Visualising image classification models and saliency maps. arXiv preprint arXiv:1312.6034, 2013.

[19] K. Sirinukunwattana, J. P. Pluim, H. Chen, X. Qi, P.-A. Heng, Y. B. Guo, L. Y. Wang, B. J. Matuszewski, E. Bruni, U. Sanchez, et al. Gland segmentation in colon histology images: The GlaS challenge contest. Medical image analysis, 35:489-502, 2017.

[20] J. R. Zech, M. A. Badgeley, M. Liu, A. B. Costa, J. J. Titano, and E. K. Oermann. Variable generalization performance of a deep learning model to detect pneumonia in chest radiographs: A cross-sectional study. PLoS medicine, 15(11):e1002683, 2018.

[21] B. Zhou, A. Khosla, A. Lapedriza, A. Oliva, and A. Torralba. Learning deep features for discriminative localization. In Proceedings of the IEEE conference on computer vision and pattern recognition, pages 2921-2929, 2016. 
medRxiv preprint doi: https://doi.org/10.1101/2020.02.27.20028845; this version posted February 29, 2020. The copyright holder for this preprint (which was not certified by peer review) is the author/funder, who has granted medRxiv a license to display the preprint in perpetuity. All rights reserved. No reuse allowed without permission.

A Generic Neural Network Approach to Infer Segmenting Classifiers for Disease-Associated Regions in Medical Images A PREPRINT

\section{Supplemental Materials: A Generic Neural Network Approach to Infer Segmenting Classifiers for Disease-Associated Regions in Medical Images}
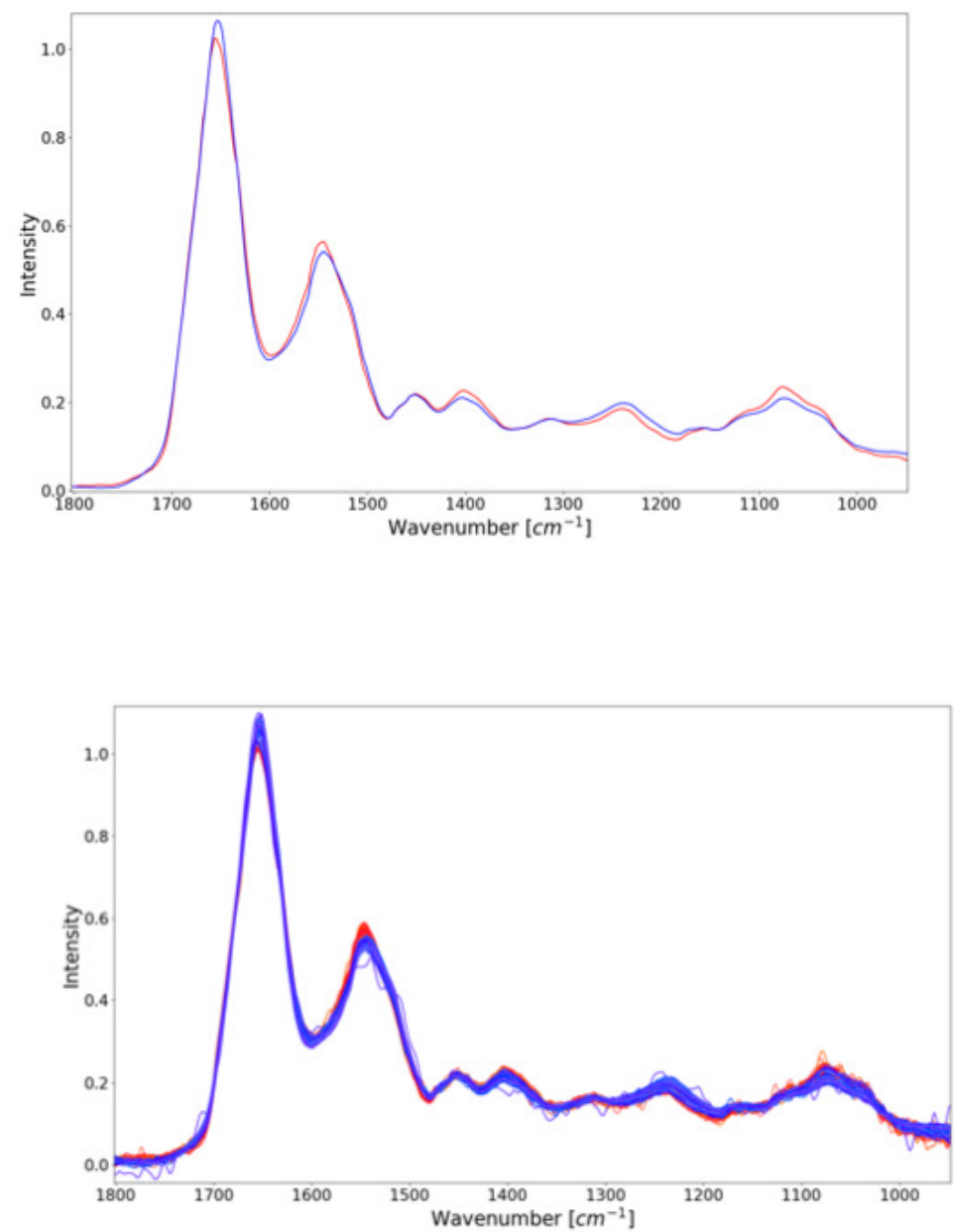

Figure S1: Spectral differences between tissue components. Top: Differences between average spectra of tumor (red) and non-tumor (specifically crypts, blue spectra) are seemingly very small. Bottom: 100 randomly sampled tumor (red) and crypt (blue) spectra indicate systematic differences between the two tissue components. In label-free digital pathology, it is well-established that these small differences are highly discriminative for differentiating many tissue components and certain disease-substypes. 
medRxiv preprint doi: https://doi.org/10.1101/2020.02.27.20028845; this version posted February 29, 2020. The copyright holder for this preprint (which was not certified by peer review) is the author/funder, who has granted medRxiv a license to display the preprint in perpetuity. All rights reserved. No reuse allowed without permission.

A Generic Neural Network Approach to Infer Segmenting Classifiers for Disease-Associated Regions in Medical Images A PREPRINT

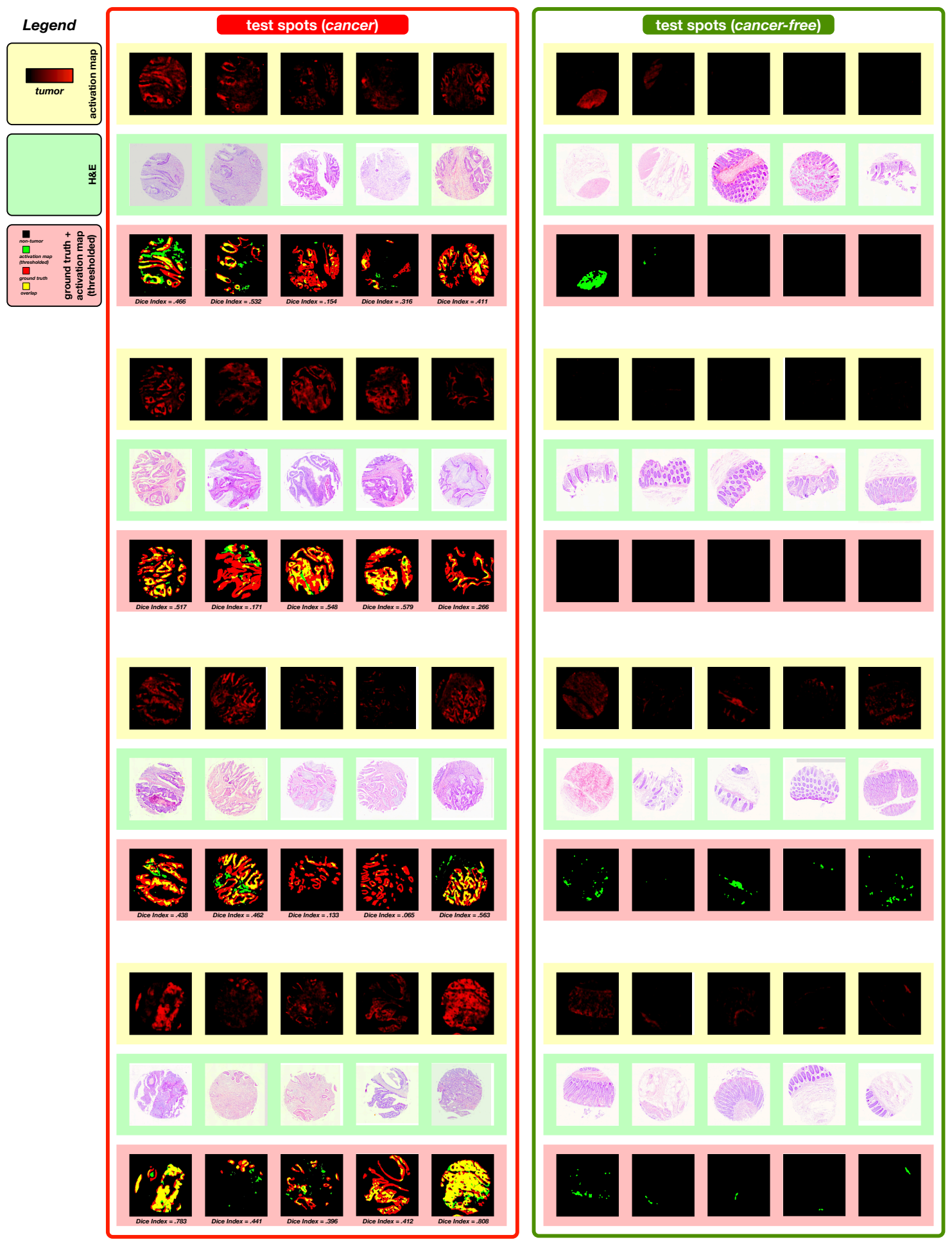

Figure S2: Activation Maps, $H \& E$ stains, and ground truth annotations obtained from the 40 spots of the class-balanced independent test set. The classifier trained on the 100 tissue microarray spots from slides $C O 1002 b$ and $C O 722$ of the training set was applied to 40 test spots from slide $B C 051111$. The identical threshold that was used to segment tumor in the validation data set $(.35 \cdot 255)$ was applied to identify tumor regions. Tumor structures are well matched in most of the cancer spots, while most of the four cancer-free spots exhibit only a low degree of activation. 
medRxiv preprint doi: https://doi.org/10.1101/2020.02.27.20028845; this version posted February 29, 2020. The copyright holder for this preprint (which was not certified by peer review) is the author/funder, who has granted medRxiv a license to display the preprint in perpetuity. All rights reserved. No reuse allowed without permission.

A Generic Neural Network Approach to Infer Segmenting Classifiers for Disease-Associated Regions in Medical Images A PREPRINT

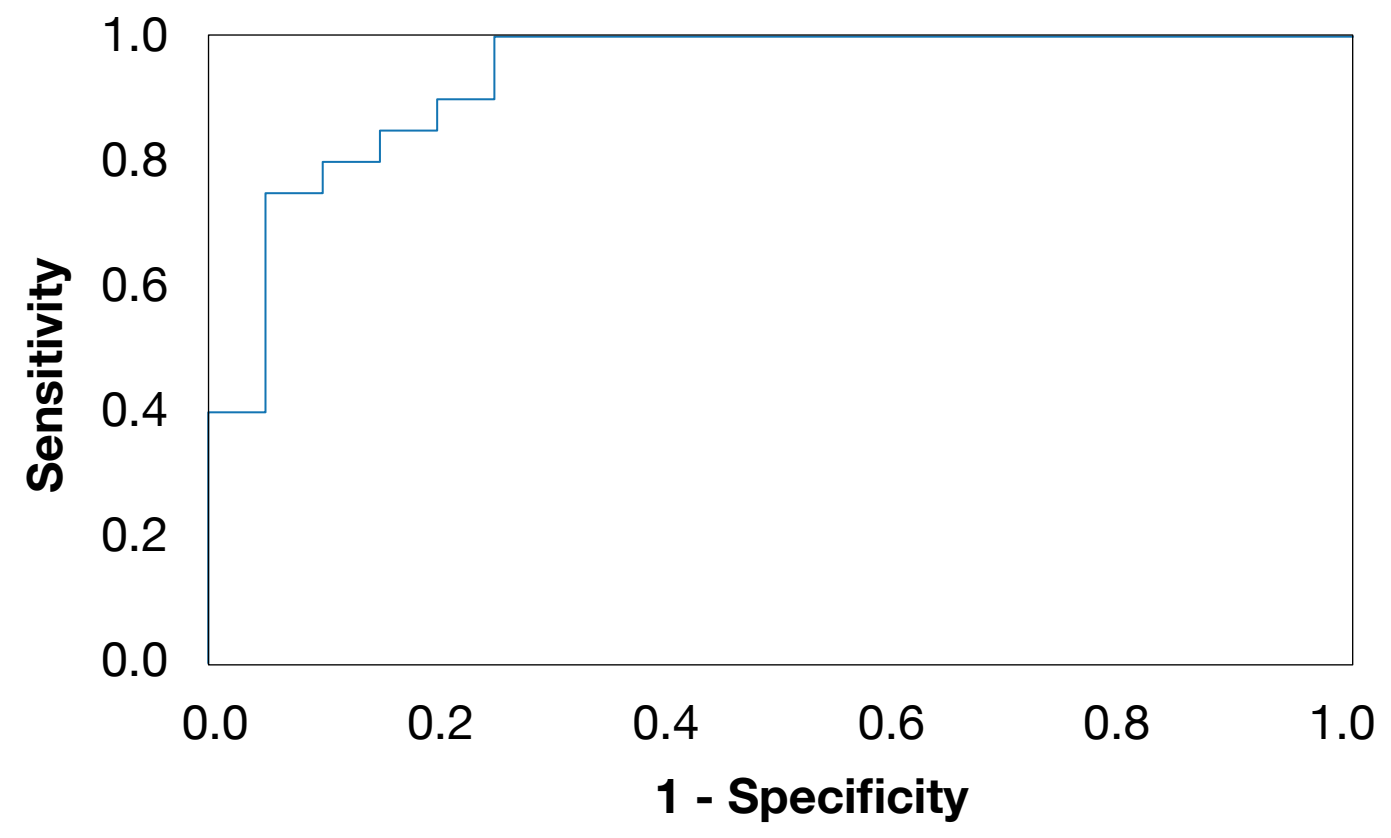

Figure S3: ROC Curve of thresholded activation maps. Activation maps of all 20 cancer and 20 cancer-free spots in the class-balanced independent test set were thresholded with $\theta=.35 \cdot 255$. Foreground pixels were considered as tumor and background pixels as non-tumor. The ROC curve was obtained by varying a secondary threshold of the ratio of tumor pixels to classify a spot as a cancer spot. The displayed ROC curve covers an AUC of $93.25 \%$. 